worms in the lawns. The crane-fly, which usually swarms in the fields of the Mansfield estate in September, has been very rare, too, this season. The dragon-fly visited us this summer for the first time.

Apropos to the records of the "rare phenomenon," such a summer aurora was observed at Rothbury, Northumberland, in the latter half of August I880.

To conclude this farrago of notes: for " non pas travaillés" in Mr. Sclater's quotation of the Prince of Canino's words (xliv. p. 518), read "n'ont. . ."

Hampstead, N.W., October 3 . J. J. WALKER.

\section{THE MOLECULAR PROCESS IN MAGNETIC INDUCTION.}

$\mathrm{M}$ AGNETIC induction is the name given by Faraday to the act of becoming magnetized, which certain substances perform when they are placed in a magnetic field. A magnetic field is the region near a magnet, or near a conductor conveying an electric current. Throughout such a region there is what is called magnetic force, and when certain substances are placed in the magnetic field the magnetic force causes them to become magnetized by magnetic induction. An effective way of producing a magnetic field is to wind a conducting wire into a coil, and pass a current through the wire. Within the coil we have a region of comparatively strong magnetic force, and when a piece of iron is placed there it may be strongly magnetized. Not all substances possess this property. Put a piece of wood or stone or copper or silver into the field, and nothing noteworthy happens ; but put a piece of iron or nickel or cobalt and at once you find that the piece has become a magnet. These three metals, with some of their alloys and compounds, stand out from all other substances in this respect. Not only are they capable of magnetic induction-of becoming magnets while exposed to the action of the magnetic field-but when withdrawn from the field they are found to retain a part of the magnetism they acquired. They all show this property of retentiveness, more or less. In some of them this residual magnetism is feebly held, and may be shaken out or otherwise removed without difficulty. In others, notably in some steels, it is very persistent, and the fact is taken advantage of in the manufacture of permanent magnets, which are simply bars of steel, of proper quality, which have been subjected to the action of a strong magnetic field. Of all substances, soft iron is the most susceptible to the action of the field. It can also, under favourable conditions, retain, when taken out of the field, a very large fraction of the magnetism that has been induced-more than ninetenths-more, indeed, than is retained by steel ; but its hold of this residual magnetism is not firm, and for that reason it will not serve as a material for permanent magnets. My purpose to-night is to give some account of the molecular process through which we may conceive magnetic induction to take place, and of the structure which makes residual magnetism possible.

When a piece of iron or nickel or cobalt is magnetized by induction, the magnetic state permeates the whole piece. It is not a superficial change of state. Break the piece into as many fragments as you please, and you will find that every one of these is a magnet. In seeking an explanation of magnetic quality we must penetrate the innermost framework of the substance-we must go to the molecules.

Now, in a molecular theory of magnetism there are two possible beginnings. We might suppose, with Poisson, that each molecule becomes magnetized when the field begins to act. Or we may adopt the theory of Weber, which says that the molecules of iron are always magnets, and that what the field does is to turn them so

'Abstract of a Friday Evening Discourse delivered at the Royal Institution on May 22, 1891, by J. A. Ewing, M.A., F.R.S., Professor of Applied Mechanics and Mechanism in the University of Cambridge. that they face more or less one way. According to this view, a virgin piece of iron shows no magnetic polarity, not because its molecules are not magnets, but because they lie so thoroughly higgledy-piggledy as regards direction that no greater number point one way than another. But when the magnetic force of the field begins to act, the molecules turn in response to it, and so a preponderating number come to face in the direction in which the magnetic force is applied, the result of which is that the piece as a whole shows magnetic polarity. All the facts go to confirm Weber's view. One fact in particular I may mention at once-it is almost conclusive in itself. When the molecular magnets are all turned to face one way, the piece has clearly received as much magnetization as it is capable of. Accordingly, if Weber's theory be true, we must expect to find that in a very strong magnetic field a piece of iron or other magnetizable metal becomes saturated, so that it cannot take up any more magnetism, however much the field be strengthened. This is just what happens: experiments were published a few years ago which put the fact of saturation beyond a doubt, and gave values of the limit to which the intensity of magnetization may be forced.

When a piece of iron is put in a magnetic field, we do not find that it becomes saturated unless the field is exceedingly strong. A weak field induces but little magnetism; and if the field be strengthened, more and more magnetism is acquired. This shows that the molecules do not turn with perfect readiness in response to the deflecting magnetic force of the field. Their turning is in some way resisted, and this resistance is overcome as the field is strengthened, so that the magnetism of the piece increases step by step. What is the directing force which prevents the molecules from at once yielding to the deflecting influence of the field, and to what is that force due? And again, how comes it that after they have been deflected they return partially, but by no means wholly, to their original places when the field ceases to act?

I think these questions receive a complete and satisfactory answer when we take account of the forces which the molecules necessarily exert on one another in consequence of the fact that they are magnets. We shall study the matter by examining the behaviour of groups of little magnets, pivoted like compass needles, so that each is free to turn except for the constraint which each one suffers on account of the presence of its neighbours.

But first let us see more particularly what happens when a piece of iron or steel or nickel or cobalt is magnetized by means of a field the strength of which is gradually augmented from nothing. We may make the experiment by placing a piece of iron in a coil, and making a current flow in the coil with gradually increased strength, noting at each stage the relation of the induced magnetism to the strength of the field. This relation is observed to be by no means a simple one: it may be represented by a curve (Fig. I), and an inspection of the curve will show that the process is divisible, broadly, into three tolerably distinct stages. In the first stage $(a)$ the magnetism is being acquired but slowly: the molecules, if we accept Weber's theory, are not responding readilythey are rather hard to turn. In the second stage $(b)$ their resistance to turning has to a great extent broken down, and the piece is gaining magnetism fast. In the third stage $(c)$ the rate of increment of magnetism falls off : we are there approaching the condition of saturation, though the process is still a good way from being completed.

Further, if we stop at any point of the process, such as $P$, and gradually reduce the current in the coil until there is no current, and therefore no magnetic field, we shall get a curve like the dotted line $P Q$, the height of $Q$ showing the amount of the residual magnetism.

If we make this experiment at a point in the first stage NO. I I 46, VOL. 44] 
(a), we shall find, as Lord Rayleigh has shown, little or no residual magnetism; if we make it at any point in the second stage $(b)$, we shall find very much residual magnetism; and if we make it at any point in the third stage (c), we shall find only a little more residual magnetism than we should have found by making the experiment at the end of stage $b$. That part of the turning of the molecules which goes on in stage $a$ contributes nothing to the residual magnetism. That part which goes on in stage $c$ contributes little. But that part of the turning which goes on in stage $b$ contributes very much.

In some specimens of magnetic metal we find a much

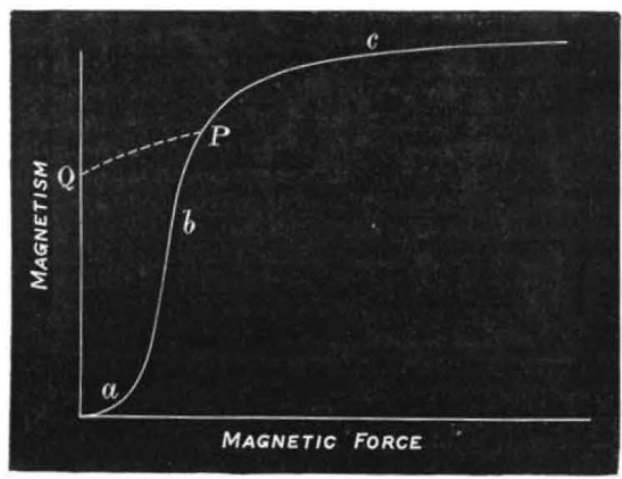

FIG. I.

sharper separation of the three stages than in others. By applying strain in certain ways it is possible to get the stages very clearly separated. Fig. 2, a beautiful instance of that, is taken from a paper by Mr. Nagaoka - one of an able band of Japanese workers who are bidding fair to repay the debt that Japan owes for its learning to the West. It shows how a piece of nickel which is under the joint action of pull and twist becomes magnetized in a growing magnetic field. There the first stage is exceptionally prolonged, and the second stage is extraordinarily abrupt.

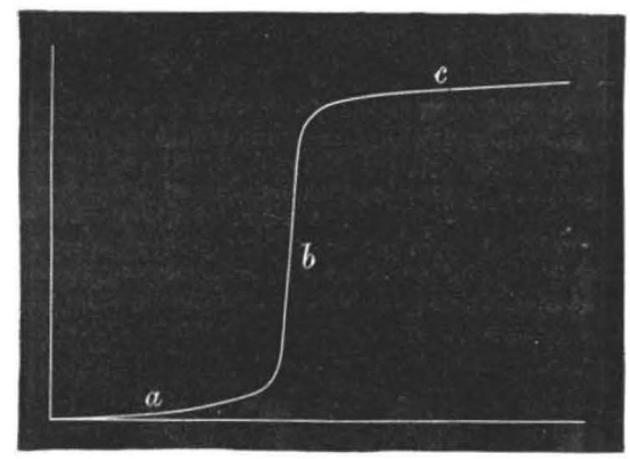

FiG. 2.

The bearing of all this on the molecular theory will be evident when we turn to these models, consisting of an assemblage of little pivoted magnets, which may be taken to represent, no doubt in a very crude way, the molecular structure of a magnetizable metal. I have here some large models, where the pivoted magnets are pieces of sheet steel, some cut into short flat bars, others into diamond shapes with pointed ends, others into shapes resembling mushrooms or umbrellas, and in these the magnetic field is produced by means of a coil of insulated wire wound on a large wooden frame below the magnets. Some of these are arranged with the pivots on a gridiron or lazy-tongs of jointed wooden bars, so that we may readily distort them, and vary the distances of the pivots from one another, to imitate some of the effects of strain in the actual solid. But to display the experiments to a large audience a lantern model will serve best. In this one the magnets are got by taking to pieces numbers of little pocket compasses. The pivots are cemented to a glass plate, through which the light passes in such a way as to project the shadows of the magnets on the screen. The magnetic force is applied by means of two coils, one on either side of the assemblage of magnets and out of the way of the light, which togetber produce a nearly uniform magnetic field throughout the whole group. You see this when I make manifest the

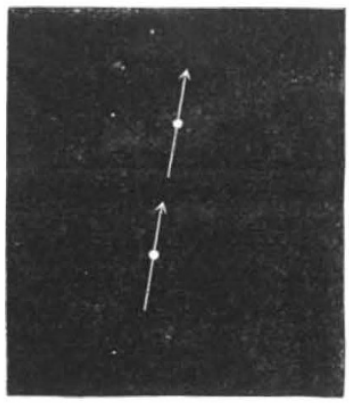

FIG. 3 .

field in a well-known fashion, by dropping iron filings on the plate.

We shall first put a single pivoted magnet on the plate. So long as no field acts it is free to point anyhow - there is no direction it prefers to any other. As soon as I apply even a very weak field it responds, turning at once into the exact direction of the applied force, for there was nothing (beyond a trifling friction at the pivot) to prevent it from turning.

Now try two magnets. I have cut off the current, so that there is at present no field, but you see at once that the pair has, so to speak, a will of its own. I may shake or disturb them as I please, but they insist on taking up a position (Fig. 3) with the north end of one as close as

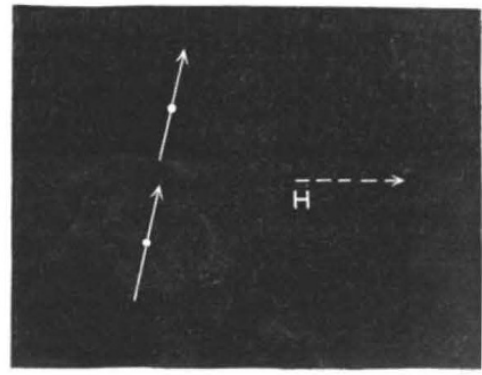

FIG. 4.

possible to the south end of the other. If disturbed they return to it: this configuration is highly stable. Watch what happens when the magnetic field acts with gradually growing strength. At first, so long as the field is weak (Fig. 4), there is but little deflection; but as the deflection increases it is evident that the stability is being lost, the state is getting more and more critical, until (Fig. 5) the tie that holds them together seems to break, and they suddenly turn, with violent swinging, into almost perfect alignment with the magnetic force $\mathrm{H}$. Now I gradually remove the force, and you see that they are slow to return, but a stage comes when they swing back, and a

NO. I I 46, VOL. 44] 
complete removal of the force brings them into the condition with which we began (Fig. 3).

If we were to picture a piece of iron as formed of a vast number of such pairs of molecular magnets, each pair far enough from its neighbours to be practically out of reach of their magnetic influence, we might deduce many of the observed magnetic properties, but not all.

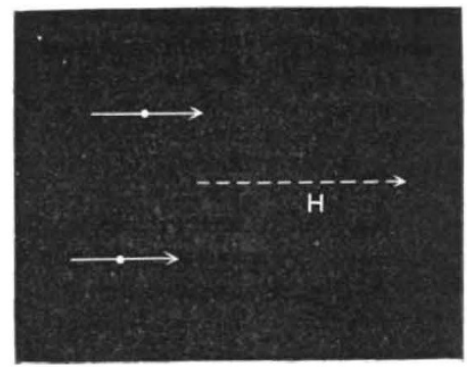

FIG. 5 .

In particular, we should not be able to account for so much residual magnetism as is actually found. To get that, the molecules must make new connections when the old ones are broken; their relations are of a kind more complex than the quasi-matrimonial one which the experiment exhibits. Each molecule is a member of a larger

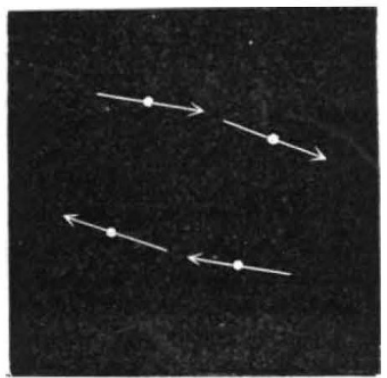

FIG. 6 .

community, and has probably many neighbours close enough to affect its conduct.

We get a better idea of what happens by considering four magnets (Fig. 6). At first, in the absence of deflecting magnetic force, they group themselves in stable pairs -in one of a number of possible combinations. Then-

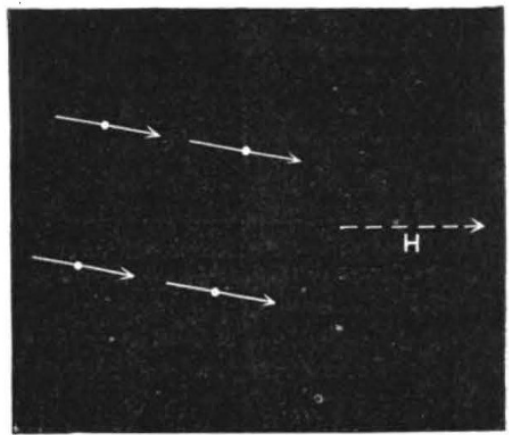

FiG. 7.

as in the former case--when magnetic force is applied, they are at first slightly deflected, in a manner that exactly tallies with what I have called the stage $a$ of the magnetizing process. Next comes instability. The original ties break up, and the magnets swing violently round; but finding a new possibility of combining (Fig. 7), they take No. I I 46 , vOL. 44$]$ to that. Finally, as the field is further strengthened, they are drawn into perfect alignment with the applied magnetic force (Fig. 8).

We see the same three stages in a multiform group (Figs. 9, 10, II). At first, the group, if it is shuffled by any casual disturbance, arranges itself at random in lines that give no resultant polarity (Fig. 9). A weak force produces no more than slight quasi-elastic deflections; a stronger force breaks up the old lines, and forms new ones

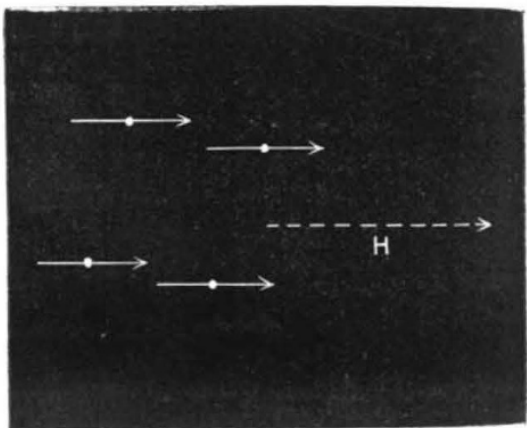

FiG. 8.

more favourably inclined to the direction of the force (Fig. 10). A very strong force brings about saturation (Fig. II).

In an actual piece of iron there are multitudes of groups lying differently directed to begin with-perhaps also different as regards the spacing of their members. Some enter the second stage while others are still in the first, and so on. Hence, the curve of magnetization does not

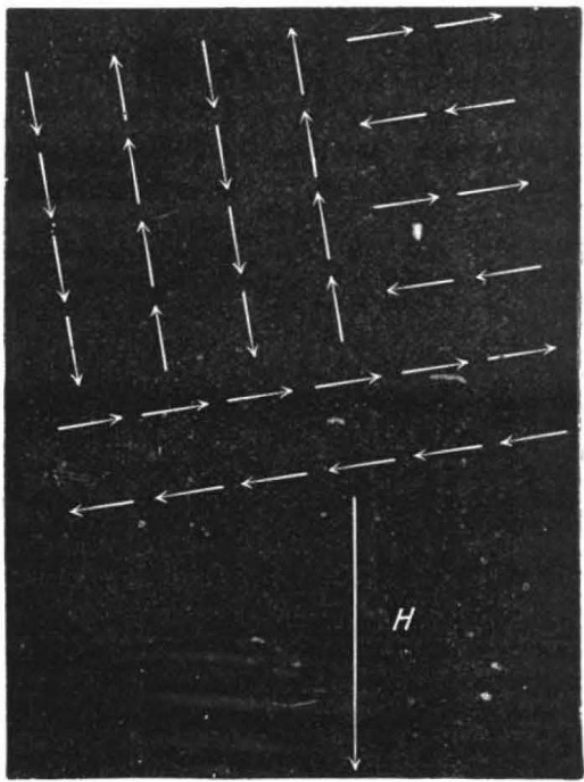

FIG. 9.

consist of perfectly sharp steps, but has the rounded outlines of Fig. I.

Notice, again, how the behaviour of these assemblages of elementary magnets agrees with what I have said about residual magnetism. If we stop strengthening the field before the first stage is passed-before any of the magnets have become unstable and have tumbled round into new plares - the small deflection simply disappears, 
and there is no residual effect on the configuration of the group. But if we carry the process far enough to have unstable deflections, the effects of these persist when the force is removed, for the magnets then retain the new

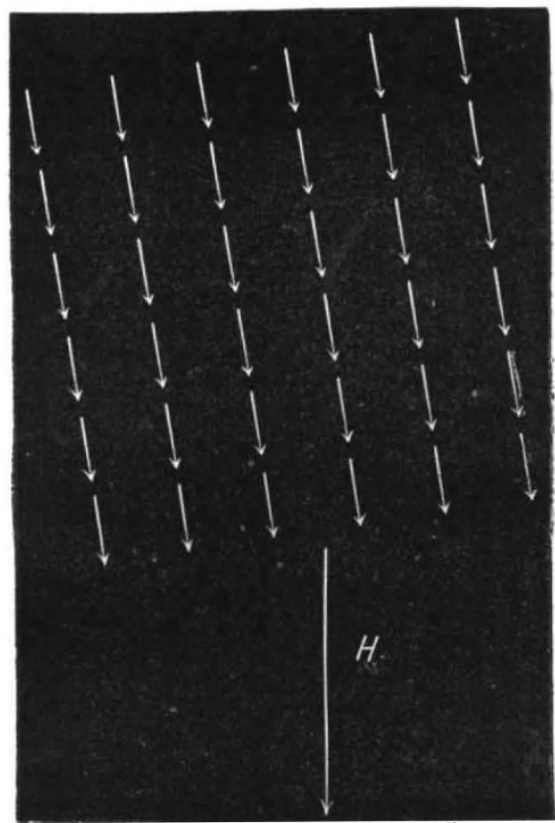

FIG. 10.

grouping into which they have fallen (Fig. 10). And again, the quasi-elastic deflections whicls go on during the third stage do not add to the residual magnetism.

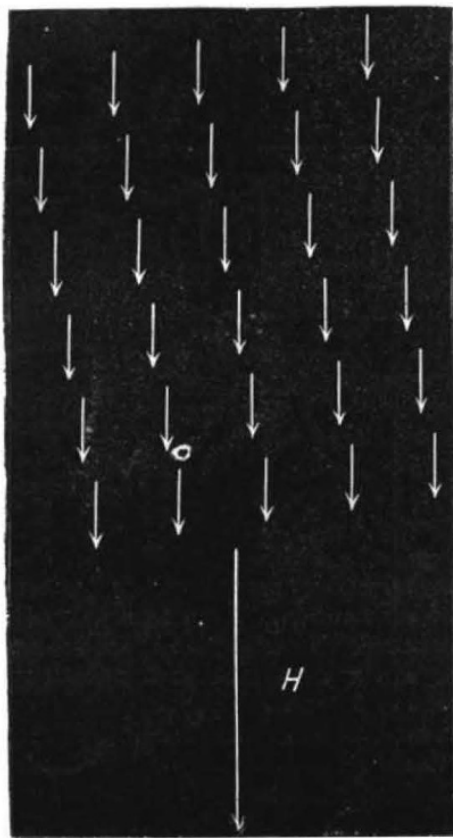

FIG. II.

Notice, further, what happens to the group if after applying a magnetic force in one direction and removing it, I begin to apply force in the opposite direction. At first there is little reduction of the residual polarity, till a NO. I I 46, VOL. 4t] stage is reached when instability begins, and then reversal occurs with a rush. We thus find a close imitation of all the features that are actually observed when iron or any of the other magnetic metals is carried through a cyclic magnetizing process (Fig. I2). The effect of any such process is to form a loo $p$ in the curve which expresses the relation of the magnetism to the magnetizing force. The changes of magnetism always lag behind the changes of magnetizing force. This tendency to lag behind is called magnetic hysteresis.

We have a manifestation of hysteresis whenever a magnetic metal has its magnetism changed in any mannet through changes in the magnetizing force, unless indeed the changes are so minute as to be confined to what I have called the first stage ( $a$, Fig. 1$)$. Residual magnetism is only a particular case of hysteresis.

Hysteresis comes in whatever be the character or cause of the magnetic change, provided it involves such deflections on the part of the molecules as make them become unstable. The unstable movements are not reversible with respect to the agent which produces them;

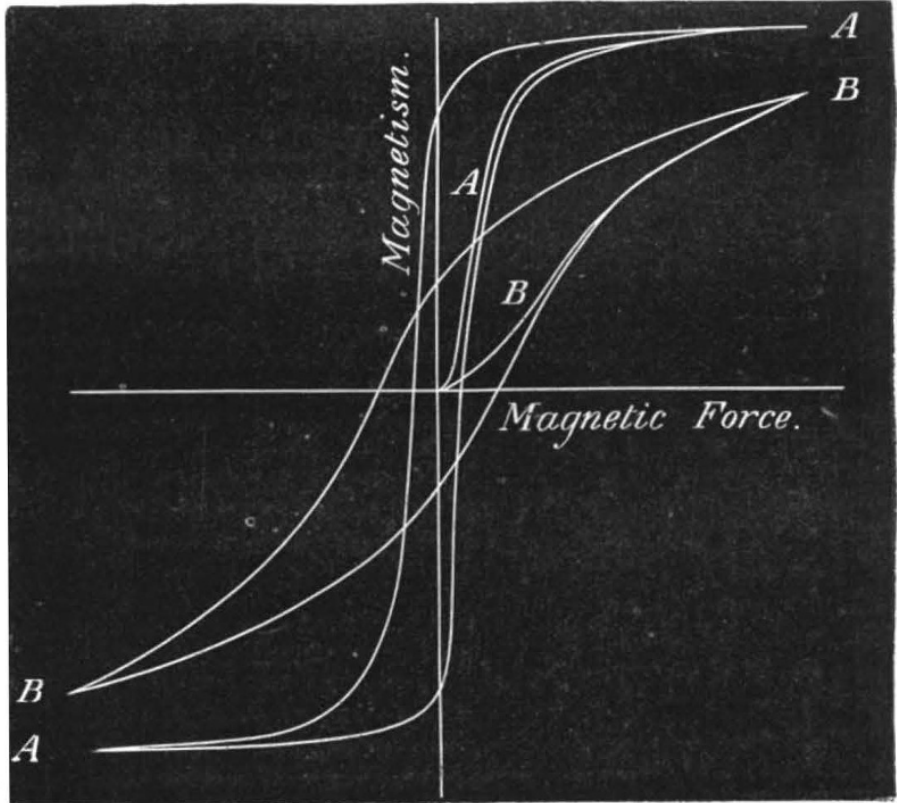

F1G. 12.-Cyclic reversal of magnetization in soft iron (AA), and in the same iron when hardened by stretching $(\mathrm{BB})$.

that is to say, they are not simply undone step by step as the agent is removed.

We know, on quite independent grounds, that when the magnetism of a piece of iron or steel is reversed, or indeed cyclically altered in any way, some work is spent in performing the operation--energy is being given to the iron at one stage, and is being recovered from it at another; but when the cycle is taken as a whole, there is a net loss, or rather a waste of energy. It may be shown that this waste is proportional to the area of the loop in our diagrams. This energy is dissipated ; that is to say, it is scattered and rendered useless : it takes the form of heat. The iron core of a transformer, for instance, which is having its magnetism reversed with every pulsation of the alternating current, tends to become hot for this very reason; indeed, the loss of energy which happens in it, in consequence of magnetic hysteresis, is a serious drawback to the efficiency of alternating-current systems of distributing electricity. It is the chief reason why they 
require much more coal to be burnt, for every unit of electricity sold, than direct-current systems require.

The molecular theory shows how this waste of energy occurs. When the molecule becomes unstable and tumbles violently over, it oscillates and sets its neighbours oscillating, until the oscillations are damped out by the eddy currents of electricity which they generate in the surrounding conducting mass. The useful work that can be got from the molecule as it falls over is less than the work that is done in replacing it during the return portion of the cycle. This is a simple mechanical deduction from the fact that the movement has unstable phases.

I cannot attempt, in a single lecture, to do more than glance at several places where the molecular theory seems to throw a flood of light on obscure and complicated facts, as soon as we recognize that the constraint of the molecules is due to their mutual action as magnets.

It has been known since the time of Gilbert that vibration greatly facilitates the process of magnetic induction. Let a piece of iron be briskly tapped while it lies in the magnetic field, and it is found to take up a large addition to its induced magnetism. Indeed, if we examine the successive stages of the process while the iron is kept vibrating by being tapped, we find that the first stage $(a)$ has practically disappeared, and there is a steady and rapid growth of magnetism almost from the very first. This is intelligible enough. Vibration sets the molecular magnets oscillating, and allows them to break their primitive mutual ties and to respond to weak deflecting forces. For a similar reason, vibration should tend to reduce the residue of magnetism which is left when the magnetizing force is removed, and this, too, agrees with the results of observation.

Perhaps the most effective way to show the influence of vibration is to apply a weak magnetizing force first, before tapping. If the force is adjusted so that it nearly but not quite reaches the limit of stage $(a)$, a great number of the molecular magnets are, so to speak, hovering on the verge of instability, and when the piece is tapped they go over like a house of cards, and magnetism is acquired with a rush. Tapping always has some effect of the same kind, even though there has been no special adjustment of the field.

And other things besides vibration will act in a similar way, precipitating the break-up of molecular groups when the ties are already strained. Change of temperature will sometimes do it, or the application or change of mechanical strain. Suppose, for instance, that we apply pull to an iron wire while it hangs in a weak magnetic field, by making it carry a weight. The first time that we put on the weight, the magnetism of the wire at once increases, of en very greatly, in consequence of the action I have just described (Fig. I 3). The molecules have been on the verge of turning, and the slight strain caused by the weight is enough to make them go. Remove the weight, and there is only a comparatively small change in the magnetism, for the greater part of the molecular turning that was done when the weight was put on is not undone when it is taken off. Reapply the weight, and you find again but little change, though there are still traces of the kind of action which the first application brought about. That is to say, there are some groups of molecules which, though they were not broken up in the first application of the weight, yield now, because they have lost the support they then obtained from neighbours that have now entered into new combinations. Indeed, this kind of action may often be traced, always diminishing in amount, during several successive applications and removals of the load (see Fig. 13), and it is only when the process of loading has been many times repeated that the magnetic change brought about by loading is just opposite to the magnetic change brought about by unloading.

Whenever, indeed, we are observing the effects of an alteration of physical condition on the magnetism of iron, we have to distinguish between the primitive effect, which is often very great and is not reversible, and the ultimate effect, which is seen only after the molecular structure has become somewhat settled through many repetitions of the process. Experiments on the effects of temperature, of strain, and so forth, have long ago shown this distinction to be exceedingly important : the molecular theory makes it perfectly intelligib!e.

Further, the theory makes plain another curious result of experiment. When we have loaded and unloaded the iron wire many times over, so that the effect is no longer complicated by the primitive action I have just described, we still find that the magnetic changes which occur while the load is being put on are not simply undone, step by step, while the load is being taken off. Let the whole load be divided into several parts, and you will see that the magnetism has two different values, in going up and in coming down, for one and the same intermediate value of the load. The changes of magnetism lag behind the changes of load : in other words, there is hysteresis in the

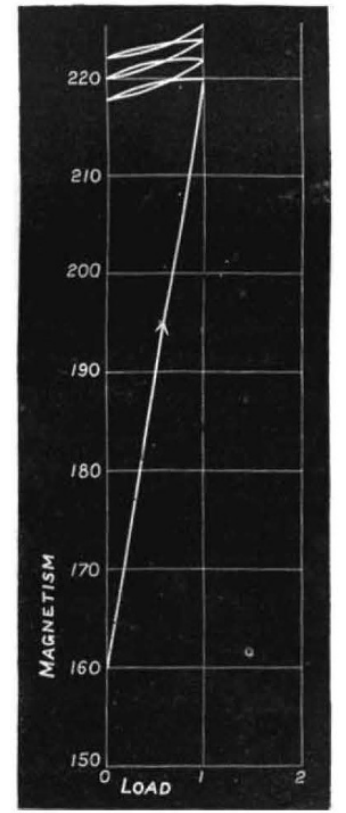

FIG. r3.-Effects of loading a soft iron wire in a constant field.

relation of the magnetism to the load (Fig. 14). This is because some of the molecular groups are every time being broken up during the loading, and re-established during the unloading, and that, as we saw already, involves hysteresis. Consequently, too, each loading and unloading requires the expenditure of a small quantity of energy, which goes to heat the metal.

Moreover, a remarkably interesting conclusion follows. This hysteresis, and consequent dissipation of energy, will also happen though there be no magnetization of the piece as a whole: it depends on the fact that the molecules are magnets. Accordingly, we should expect to find, and experiment confirms this (see Phil. Trans., I 885 , p. 6I4), that if the wire is loaded and unloaded, even when no magnetic field acts and there is no magnetism, its physical qualities which are changed by the load will change in a manner involving hysteresis. In particular, the length will be less for the same load during loading than during unloading, so that work may be wasted in every cycle of loads. There can be no such thing as perfect elasticity in a magnetizable metal, unless, indeed, the range of the strain is so very narrow that none of the

NO. I I 46, VOL. 44] 
molecules tumble through unstable states. This may have something to do with the fact, well known to engineers, that numerous repetitions of a straining action, so slight as to be safe enough in itself, have a dangerous effect on the structure of iron or steel.

Another thing on which the theory throws light is the phenomenon of time-lag in magnetization. When a piece of iron is put into a steady magnetic field, it does not take instantly all the magnetism that it will take if time be allowed. There is a gradual creeping up of the magnetism, which is most noticeable when the field is weak and when the iron is thick. If you will watch the manner in which a group of little magnets breaks up when a magnetic force is applied to it, you will see that the process is one that takes time. The first molecule to yield is some outlying one which is comparatively unattachedas we may take the surface molecules in the piece of iron

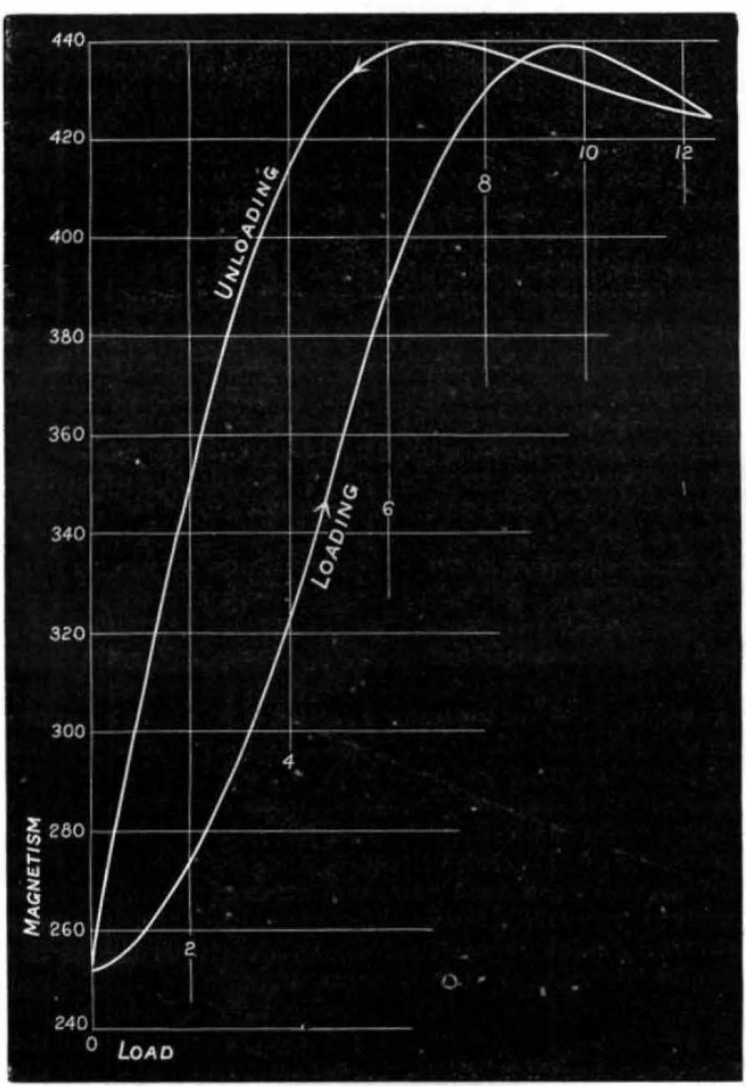

FIG. 14.-Cycle of loading and unloading.

to be. It falls over, and then its neighbours, weakened by the loss of its support, follow suit, and gradually the disturbance propagates itself from molecule to molecule throughout the group. In a very thin piece of iron-a fine wire, for instance - there are so many surface molecules, in comparison with the whole number, and consequently so many points which may become origins of disturbance, that the breaking up of the molecular communities is too soon over to allow much of this kind of lagging to be noticed.

Effects of temperature, again, muy be interpreted by help of the molecular theory. When iron or nickel or cobalt is heated in a weak magnetic field, its susceptibility to magnetic induction is observed to increase, until a stage is reached, at a rather high temperature, when the magnetic quality vanishes almost suddenly and almost completely. NO. I I 46, vOL. 44]
Fig. I 5 , from one of Hopkinson's papers, shows what is observed as the temperature of a piece of steel is gradually raised. The sudden loss of magnetic quality occurs when the metal has become red-hot; the magnetic quality is recovered when it cools again sufficiently to cease to glow. Now, as regards the first effect-the increase of susceptibility with increase of temperature--I think that is a consequence of two independent effects of heating. The structure is expanded, so that the molecular centres lie further apart. But the freedom with which the molecules obey the direction of any applied magnetic force is increased not by that only, but perhaps even more by their being thrown into vibration. When the field is wea's, heating consequently assists magnetization, sometimes very greatly, by hastening the passage from stage $a$ to stage $b$ of the magnetizing process. And it is at least a conjecture worth consideration whether the sudden loss of magnetic quality at a higher temperature is not due to the vibrations becoming so violent as to set the molecules spinning, when, of course, their polarity would be of no avail to produce magnetization. We know, at all events, that when the change from the magnetic to the nonmagnetic state occurs, there is a profound molecular change, and heat is absorbed which is given out again when the reverse change takes place. In cooling from a red heat, the iron actually extends at the moment when this change takes place (as was shown by Gore), and so much heat is given out that (as Barrett observed) it re-

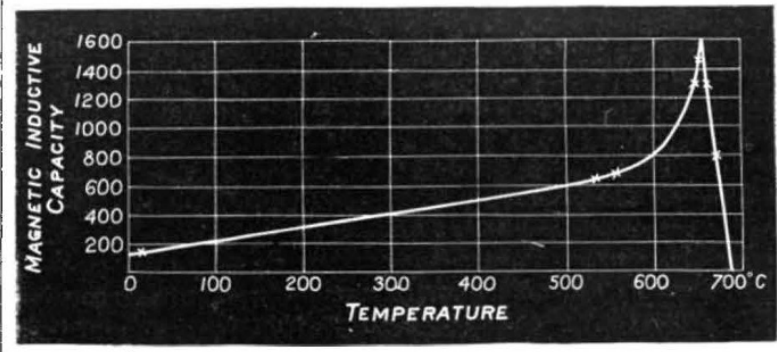

FIG. 15, - Relation of magnetic inductive capacity to teniperature in hard steel (Hopkinson).

glows, becoming brightly red, though, just before the change, it had cooled so far as to be quite dull. [Experiment, exhibiting retraction and re-glow in cooling, shown by means of a long iron wire, heated to redness by the electric current.] The changes which occur in iron and steel about the temperature of redness are very complex, and I refer to this as only one possible direction in which a key to them may be sought. Perhaps the full explanation belongs as much to chemistry as to physics.

An interesting illustration of the use of these models has reached me, only to-day, from New York. In a paper just published in the Electrical World (reprinted in the Electrician for May 29, 1891), Mr. Arthur Hoopes supports the theory I have laid before you by giving curves which show the connection, experimentally found by him, between the resultant polarity of a group of little pivoted magnets and the strength of the magnetic field, when the field is applied, removed, reversed, and so on. I shall draw these curves on the screen, and rough as they are, in consequence of the limited number of magnets, you see that they succeed remarkably well in reproducing the features which we know the curves for solid iron to possess.

It may, perbaps, be fairly claimed that the models whose behaviour we have been considering have a wider application in physics than merely to elucidate magnetic processes. The molecules of bodies may have polarity which is not magnetic at all-polarity, for instance, due to static electrification-under which they group themselves in 
stable forms, so that energy is dissipated whenever these are broken up and rearranged. When we strain a solid body beyond its limit of elasticity, we expend work irrecoverably in overcoming, as it were, internal friction. What is this internal friction due to but the breaking and making of molecular ties? And if internal friction, why atot also the surface friction which causes work to be spent when one body rubs upon another? In a highly suggestive passage of one of his writings, ${ }^{1}$ Clerk Maxwell threw out the hint that many of the irreversible processes of physics are due to the breaking up and reconstruction of molecular groups. The models help us to realize Maxwell's notion, and, in studying them to-night, I think we may claim to have been going a step or two forward where that great leader pointed the way.

\section{THE SUN'S MOTION IN SPACE.}

$S C I E N C E$ needed two thousand years to disentangle the earth's orbital movement from the revolutions of the other planets, and the incomparably more arduous problem of distinguishing the solar share in the confused multitude of stellar displacements first presented itself as possibly tractable little more than a century ago. In the lack for it as yet of a definite solution there is, then, no ground for surprise, but much for satisfaction in the large measure of success attending the strenuous attacks of which it has so often been made the object.

Approximately correct knowledge as to the direction and velocity of the sun's translation is indispensable to a profitable study of sidereal construction ; but apart from some acquaintance with the nature of sidereal construction, it is difficult, if not impossible, of attainment. One, in fact, presupposes the other. To separate a common element of motion from the heterogeneous shiftings upon the sphere of three or four thousand stars is a task practicable only under certain conditions. To begin with, the proper motions investigated must be established with general exactitude. The errors inevitably affecting them must be such as pretty nearly, in the total upshot, to neutralize one another. For should they run mainly in one direction, the result will be falsified in a degree enormously disproportionate to their magnitude. The adoption, for instance, of a system of declinations as much as $I^{\prime \prime}$ of arc astray, might displace to the extent of $10^{\circ}$ north or south the point fixed upon as the apex of the sun's way (see L. Boss, Astr. Four., No. 213). Risks on this score, however, will become less formidable with the further advance of practical astronomy along a track definable as an asymptote to the curve of ideal perfection.

Besides this obstacle to be overcome, there is another which it will soon be possible to evade. Hitherto, inquiries into the solar movement have been hampered by the necessity for preliminary assumptions of some kind as to the relative distances of classes of stars. But all such assumptions, especially when applied to selected lists, are highly insecure ; and any fabric reared upon them must be considered to stand upon treacherous ground. The spectrographic method, however, here fortunately comes into play. "Proper motions" are only angular velocities. They tell nothing as to the value of the perspective element they may be supposed to include, or as to the real rate of going of the bodies they are attributed to, until the size of the sphere upon which they are measured has been otherwise ascertained. But the displacements of lines in stellar spectra give directly the actual velocities relative to the earth of the observed stars. The question of their distances is, therefore, at once eliminated. Now the radial component of stellar motion is mixed up, precisely in the same way as the

" "Encyc. Brit.," Art. "Constitution of Bodies."

NO. I I 46, vOL. 44] tangential component, with the solar movement; and since complete knowledge of it, in a sufficient number of cases, is rapidly becoming accessible, while knowledge of tangential velocity must for a long time remain partial or uncertain, the advantage of replacing the discussion of proper motions by that of motions in line of sight is obvious and immediate. And the admirable work carried on at Potsdam during the last three years will soon afford the means of doing so in the first, if only a preliminary investigation of the solar translation based upon measurements of photographed stellar spectra.

The difficulties, then, caused either by inaccuracies in star-catalogues or by ignorance of star-distances, may be overcome; but there is a third, impossible at present to be surmounted, and not without misgiving to be passed by. All inquiries upon the subject of the advance of our system through space start with an hypothesis most unlikely to be true. The method uniformly adopted in them - and no other is available-is to treat the inherent motions of the stars (their so-called motus peculiares) as pursued indifferently in all directions. The steady drift extricable from them by rules founded upon the science of probabilities is presumed to be solar motion visually transferred to them in proportions varying with: their remoteness in space, and their situations on the sphere. If this presumption be in any degree baseless, the result of the inquiry is pro tanto falsified. Unless the deviations from the parallactic line of the stellar motions balance one another on the whole, their discussion may easily be as fruitless as that of observations tainted with systematic errors. It is scarcely, however, doubtful that law, and not chance, governs the sidereal revolutions. The point open to question is whether the workings of law may not be so exceedingly intricate as to produce a grand sum-total of results which, from the geometrical side, may justifiably be regarded as casual.

The search for evidence of a general plan in the wanderings of the stars over the face of the sky has so far proved fruitless. Local concert can be traced, but no widely-diffused preference for one direction over any other makes itself definitely felt. Some regard, nevertheless, must be paid by them to the plane of the Milky Way ; since it is altogether incredible that the actual construction of the heavens is without dependence upon the method of their revolutions.

The apparent anomaly vanishes upon the consideration of the profundities of space and time in which the fundamental design of the sidereal universe lies buried. Its composition out of an indefinite number of partial systems is more than probable ; but the inconceivable leisureliness with which their mutual relations develop renders the harmony of those relations inappreciable by short-lived terrestrial denizens. "Proper motions," if this be so, are of a subordinate kind; they are indexes simply to the mechanism of particular aggregations, and have no definable connection with the mechanism of the whole. No considerable error may then be involved in treating them, for purposes of calculation, as indifferently directed ; and the elicited solar movement may genuinely represent the displacement of our system relative to its more immediate stellar environment. This is perhaps the utmost to be hoped for until sidereal astronomy has reached another stadium of progress.

Unless, indeed, effect should be given to Clerk Maxwell's suggestion for deriving the absolute longitude of the solar apex from observations of the eclipses of Jupiter's satellites (Proc. Roy. Soc., vol. xxx. p. rog). But this is far from likely. In the first place, the revolutions of the Jovian system cannot be predicted with anything like the required accuracy. In the second place, there is no certainty that the postulated phenomena have any real existence. If, however, it be safe to assume that the solar system, cutting its way through space, virtually raises an ethereal counter-current, and if 